\title{
Smart Diet Planner for Patient
}

\author{
Aishwarya Pratapwar ${ }^{1}$, Mansi Darekar ${ }^{2}$, Priyanka Uttarwar ${ }^{3}$, Durgeshwari Naikwade ${ }^{4}$, \\ Prof. V. S Vishnupriya ${ }^{5}$ \\ Student, Comp Dept., Dr. D. Y. Patil College of Engineering, Pune, India $a^{1,2,3,4^{*}}$ \\ Guide, Comp Dept., Dr. D. Y. Patil College of Engineering, Pune, India ${ }^{5}$
}

\begin{abstract}
Diet problem is about finding the best proportion of food ingredients which could cover all daily nutrient needs with affordable cost. So in this paper to recommend the quantity of every ingredients food for a normal human or specific diet patient.to sense blood pressure, sugar level, heart rate, temperature. In order to obtain relevant information on food intake this system will check the data sensed by sensors and if any improvement is required in diet then accordingly the diet will get ordered from a smartphone application. All these techniques are non - invasive meaning the usage doesn't depend on taking out blood from the body but uses sensors to compute all the 4 parameters.
\end{abstract}

Keywords: Health Monitoring, Smart Phone, Diet Plan

\section{INTRODUCTION}

In that Various technological and standardization aspects have to be considered while designing a system in healthcare domain. In the system we propose sensors will sense the data and accordingly our diet will be scheduled. The sensed data of an individual will be sent to an application that will compare the received data with defined standard levels. If the compared results lead to any deficiency then the diet will be planned. In the system sensors are used to get data from the user's body and then the information is sent to the cloud with the help of Arduino and then the cloud is interfaced with mobile application and then all the information is shown in mobile application and then if there is any variation in range the order is placed at the grocery website. The operating system that is used in Arduino is ArdOS.

The compatibility of the system will be with android systems only.

i) The activities performed by user will include register and login. While registering all the information related to user like age, weight, etc. will be filled up initially, so this becomes one time activity.

ii) All the information of a user will be stored in the cloud.

iii) Data from sensors will be sent to Arduino Uno. Then Arduino forwards data to cloud.

iv) Then data is further transferred to mobile application.

v) Comparison between the range defined and new values takes place at cloud.

vi) If the comparison shows fluctuation of values out of the range then the person is tagged as unfit.

vii) So the user will receive a notification consisting of the sensed values and the diet suggested to him. Also it will ask for users permission whether he wants to order the food continued in diet.

viii) User can exit the app or he can go through the history of data stored related to his health.

\section{LITERATURE SURVEY}

\section{Wireless Wearable Photoplethysmography Sensors for Continuous Blood Pressure Monitoring}

Transmit data to a PC or mobile device via Bluetooth and enable continuous monitoring of BP.A $4 \mathrm{~cm}$ length sensor patch which integrates ECG and PPG sensors. By attaching the patch to the sternum, it can capture the pulse wave transit time (PWTT) and estimate the BP.

\section{AutoDietary: A Wearable Acoustic Sensor System for Food Intake Recognition in Daily LifeYin Bi, Mingsong} Lv, Chen Song, Wenyao Xu, Nan Guan, Member, IEEE, and Wang Yi, Fellow, IEEE

Nutrition-related diseases are nowadays a main threat to human health and pose great challenges to medical care. A crucial step to solve the problems is to monitor the daily food intake of a person precisely and conveniently. For this purpose, we present AutoDietary, a wearable system to monitor and recognize food intakes in daily life. An embedded 
Vol. 8, Issue 1, January 2019

hardware prototype is developed to collect food intake sensor data, which is highlighted by a high-fidelity microphone worn on the subject's neck to precisely record acoustic signals during eating in a noninvasive manner. The acoustic data are preprocessed and then sent to a smartphone via Bluetooth, where food types are recognized. In particular, we use hidden Markov models to identify chewing or swallowing events, which are then processed to extract their time/frequency-domain and nonlinear features. A lightweightdecision-tree-based algorithm is adopted to recognize the type of food. We also developed an application on the smart phone, which aggregates the food intake recognition results in a user-friendly way and provides suggestions on healthier eating, such as better eating habits or nutrition balance. Experiments show that the accuracy of food-type recognition by AutoDietary is $84.9 \%$, and those to classify liquid and solid food intakes are up to $97.6 \%$ and $99.7 \%$, respectively. To evaluate real-life user experience, we conducted a survey, which collects rating from 53 participants on wear comfort and functionalities of AutoDietary. Results show that the current design is acceptable to most of the users.

Mobile-Based Expert System For Human Diet Planning Using Optimum Neighbor Marji1, Dian Eka Ratnawati2 Informatics Engineering, Computer Science Faculty Brawijaya University Malang, East Java, Indonesia

This research proposes an expert system method torecommend the quantity of every ingredients food for a normal human or specific diet patient. Our proposed method initial state was 100 pairs of generated random value. Afterward, the pair of value which contains minimum error rate was chosen. Our proposed method uses the generated optimum neighbor as the recommendation solution. Our proposed method was implemented as an android application, named Slim Line. Based on the experiment, Slim Line able to compose the food ingredients quantity with the macronutrient needs in the range about $25 \%$ above or below nutrition needs

\section{EXISTING SYSTEM}

\section{WEIGHT LOSS \& DIET TRACKER:-}

Lose weight with your personal diet plan. Take our test to discover which food planner is the right one for you. From keto recipes to low carb \& everything in between, we have the right diet tracker for your healthy eating \& weightlossgoals.

\section{MEAL PLANNER \& RECIPES:-}

Healthy recipes help you stick to your plan. Stuck in a keto diet rut? Explore keto recipes to beat the sugar cravings or search keto recipes for snacks. Can't decide what to have? Check out the meal planner for keto diet ideas. Healthy eating is easier than you think!

\section{HEALTH TRACKER \& FOOD PLANNER:-}

Calorie counter \& food tracker functions help you see your daily nutritional values at a glance. Did a workout today? Go premium \& sync Lifesum with FitBit, Runkeeper, Moves \& other fitness apps.

\section{PROPOSE SYSTEM}

In Proposed system we are analysing the Smart diet for patient. . In our system the user first login to the system then the user with the help of sensor senses the data like Blood pressure, glucose level, Heart rate, Temperature and then all these sensors are connected to theArduino with the help of the breadboard and wires and then all the data is sent to cloud AWS with thehelp of ESP8266. Here ESP66 is the Wi-Fi module which is used for the connection with the internet. All the user details like the Name, Height, Weight, Age, Blood Group, Health information etc. are stored inside the cloud AWS.

The Cloud AWS performs functions like authentication, user subscription, publishing of data and comparing the observed values with the standard values. The Cloud AWS has the dynamo DB database to store information of user. or deny the order and if accepted the order is placed and if the user denies then the changes are made in the diet accordingly. 


\section{International Journal of Advanced Research in Computer and Communication Engineering}

Vol. 8, Issue 1, January 2019

\section{SYSTEM ARCHITECTURE}

In System architecture can register the application and registration is successful then login the application. Then with the help of Sensor they can sense the Heart rate, glucose level and blood pressure. Then this sensor are connected to Arduino. The information is send to the cloud using Wi-Fi that is ESP8266.

Then using application the user information is save in data base.

Following diagram is our system's architecture diagram:

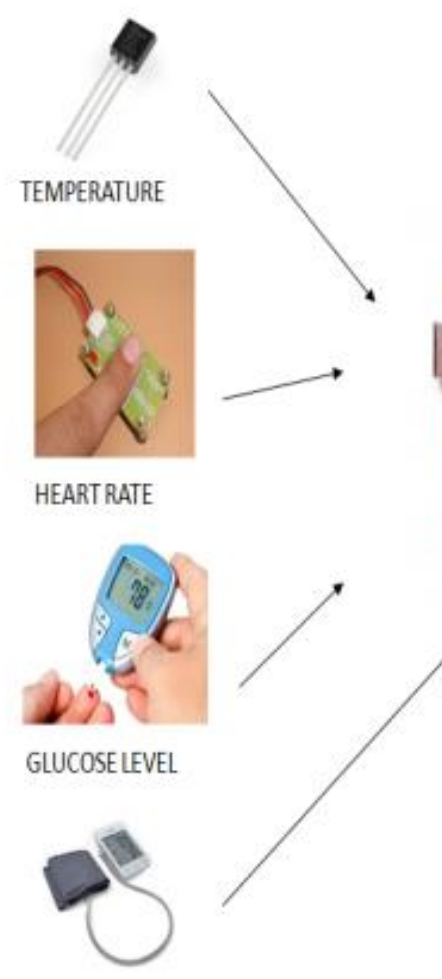

BLOOD PRESSURE
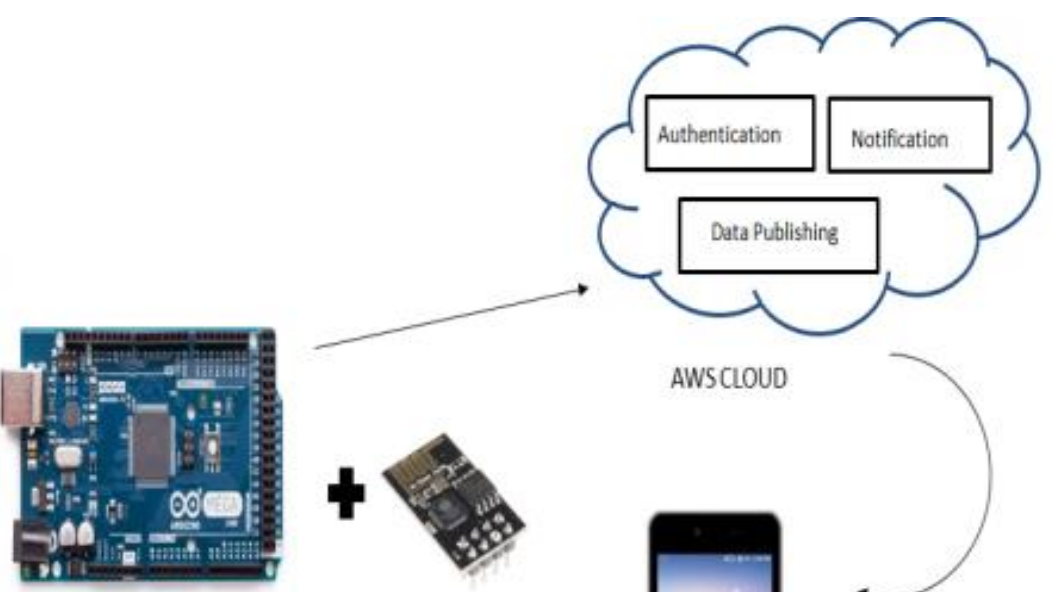

ESP 8266

ARDUINO Uno
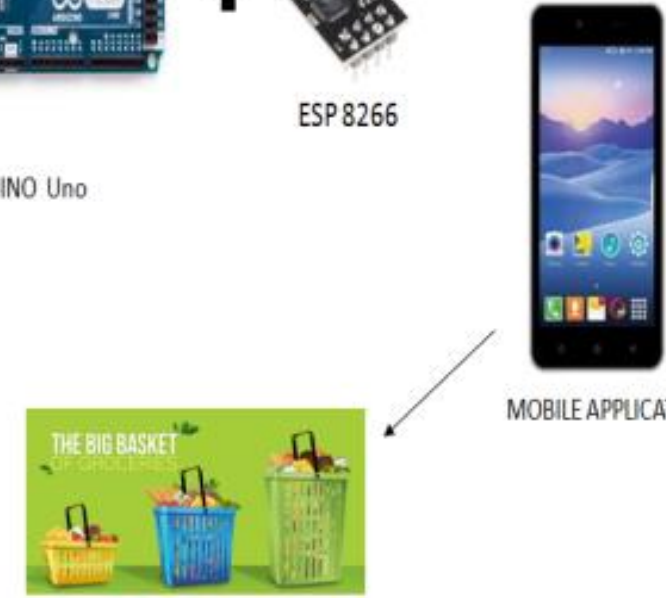

MOBILEAPPUCATION

BIG BASKET

Figure 1: system architecture

\section{METHODOLOGIES}

In Android application user information is save in database. Sensor can check the Glucose level, blood pressure and heart rate. So this information can be sense using this sensor like Arduino, Temperature sensor, Wi-Fi moduleESP8266.

Arduino:-It is an open-source electronics platform based on easy-to-use hardware and software. Arduino boards are read inputs - light on a sensor and turn it into an output - activating a motor.

Wi-FiESP8266:-It is a Wi-Fi microchip with microcontroller capability. This small module allows microcontrollers to connect to a Wi-Fi network and make simple TCP/IP connections using commands.

Temperature Sensor:- A temperature sensor is a device, that provides for temperature measurement to electrical signal. A thermocouple $(\mathrm{T} / \mathrm{C})$ is made from two dissimilar metals that generate electrical voltage in direct proportion to changes in temperature. 
Vol. 8, Issue 1, January 2019

VII. DATA FLOW DIAGRAM

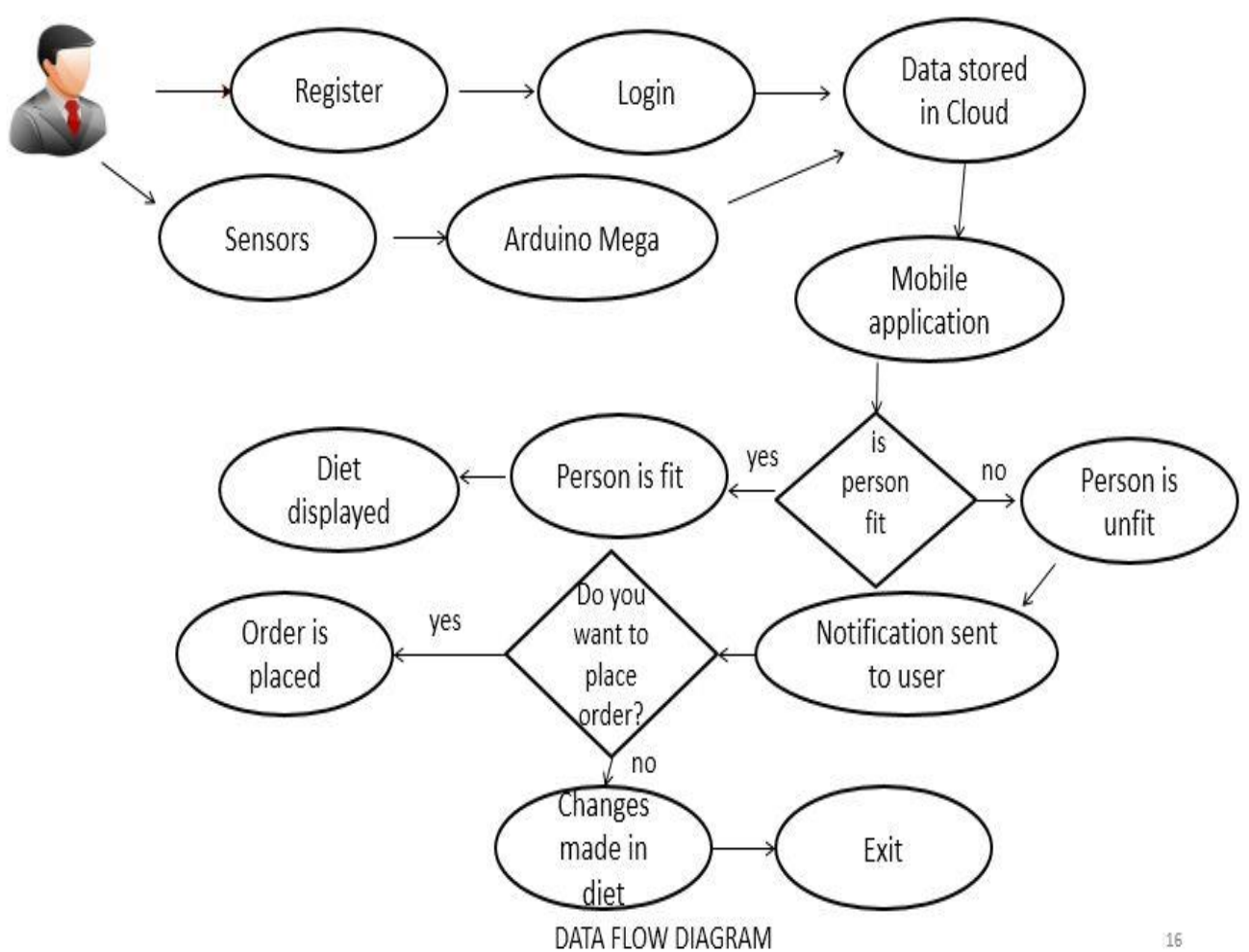

Figure2. Data Flow Diagram

\section{CONCLUSION}

Since continuous monitoring is growing requirement to reduce health risks, it is important to propose a system that maintains a person's daily diet. This system can be further improved by directly including required diet in the weekly grocery list. Also in future it may include checking the availability of required food in the fridge and notify the person about it. Using mobile applications to track different health and wellness parameters can benefit both patients and physicians. It can minimize the need for travel and give the physician the possibility to be keep track of the patients' health condition. Patients have reported that seeing their health information has prompted a change in their behaviour and therefore could have a positive influence on their lifestyle and could reduce the risk of developing age related chronic disease

\section{REFERRENCES}

[1]. A Multi-Sensor Platform for Monitoring Diabetic Peripheral Neuropathy.

[2]. Energy-Efficient Long-term Continuous Personal Health Monitoring.

[3]. Wireless Wearable Photo plethysmography Sensors for Continuous Blood Pressure Monitoring.

[4]. S. R. M. S. Baki, Z. M. A. Mohd, I. M. Yassin, A. H. Hasliza, and A. Zabidi, "Non-destructive classification of watermelon ripeness using melfrequency cepstrum coefficients and multilayer perceptrons,"in Proc. IJCNN, Jul. 2010, pp. 1-6

[5]. O. Amft and G. Troster, "On-body sensing solutions for automatic dietary monitoring," IEEE Pervasive Comput., vol. 8, no. 2, pp. 62-70, Apr./Jun. 2009.

[6]. E. S. Sazonov and S. Schuckers, "The energetics of obesity: A review: Monitoring energy intake and energy expenditure in humans," IEEE Eng.Med. Biol. Mag., vol. 29, no. 1, pp. 31-35, Jan./Feb. 2010.

[7]. S. Päßler, M. Wolff, and W.-J. Fischer, "Food intake monitoring: An acoustical approach to automated food intake activity detection and classification of consumed food," Physiol. Meas., vol. 33, no. 6,pp. 1073-1093, 2012.

[8]. K. Sato and T. Nakashima, "Human adult deglutition during sleep,” Ann. Otol., Rhinol. Laryngol., vol. 115, no. 5, pp. 334-339, 2006.

[9]. L. Mahan, S. Escott-Stump, J. Raymond, and M. Krause, \& the nutrition care process, 13th ed. St. Louis: Elsevier/Saunders, 2012. 